\title{
Revealing crystalline domains in a mollusc shell "single-crystalline" prism
}

\section{Supplementary Information}

F. Mastropietro ${ }^{1}$, P. Godard ${ }^{1 \dagger}$, M. Burghammer ${ }^{2}$, C. Chevallard ${ }^{3}$, J. Daillant $^{4}$, J. Duboisset $^{1}$,

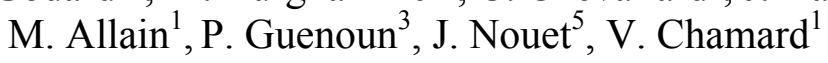

\author{
Affiliations \\ ${ }^{1}$ Aix-Marseille Univ, CNRS, Centrale Marseille, Institut Fresnel, Marseille, France. \\ ${ }^{2}$ European Synchrotron Radiation Facility, F-38043 Grenoble Cedex, France \\ ${ }^{3}$ NIMBE, CEA, CNRS, Université Paris-Saclay, CEA Saclay 91191 Gif-sur-Yvette Cedex, \\ France. \\ ${ }^{4}$ Synchrotron SOLEIL, Gif-sur-Yvette Cedex, France \\ ${ }^{5}$ GEOPS, Univ. Paris-Sud, CNRS, Université Paris-Saclay, 91405 Orsay, France \\ Correspondence to: V. Chamard, virginie.chamard@fresnel.fr \\ †Now at: Institut P', CNRS, Université de Poitiers, ENSMA, Futuroscope Chasseneuil, \\ France
}

This document contains the supplementary text and Supplementary Figures 1-8 
In the following document, we present additional experiments and details, aiming at describing the crystalline properties of the biomineral prismatic structure of Pinctada margaritifera at various scales. Those results were used as a support to the analysis presented in the main text. For the sake of clarity, the experimental results are ordered according to their capability to provide detailed direct-space and reciprocal-space information: the successive reported results correspond to increased capabilities regarding the spatial resolution and/or the reciprocal-space resolution. It results in a zoom-in view of the biomineral crystalline structure along the mesoscale.

\section{1 - Single crystalline behaviour of the Pinctada margaritifera prisms}

Prior to the Bragg ptychography experiment, a micro-diffraction experiment was performed in order to evaluate the overall crystalline quality of the prims at the shell border (Supplementary Figure 1). For these micro-diffraction measurements, the beam configuration was the same as for the Bragg ptychography experiment (see Methods). In addition, a large field-of-view detector (Frelon camera) was placed at about $7 \mathrm{~cm}$ from the sample, allowing recording simultaneously multiple Brag peaks. Those latter appear as soon as Bragg diffraction conditions are met, either during the spatial scanning of the shell across the beam (using scanning step sizes of about $2 \mu \mathrm{m}$, for a full scanned area of $100 \times 100 \mu \mathrm{m}^{2}$ ) or during the rocking curve acquisition (with angular range of $25^{\circ}$ ). Typical diffraction patterns are presented in Supplementary Figure 1. Those measurements evidence, at these scales, the homogeneous behaviour of the individual prims with respect to crystalline properties, as well as their individual single-crystalline like behaviour.

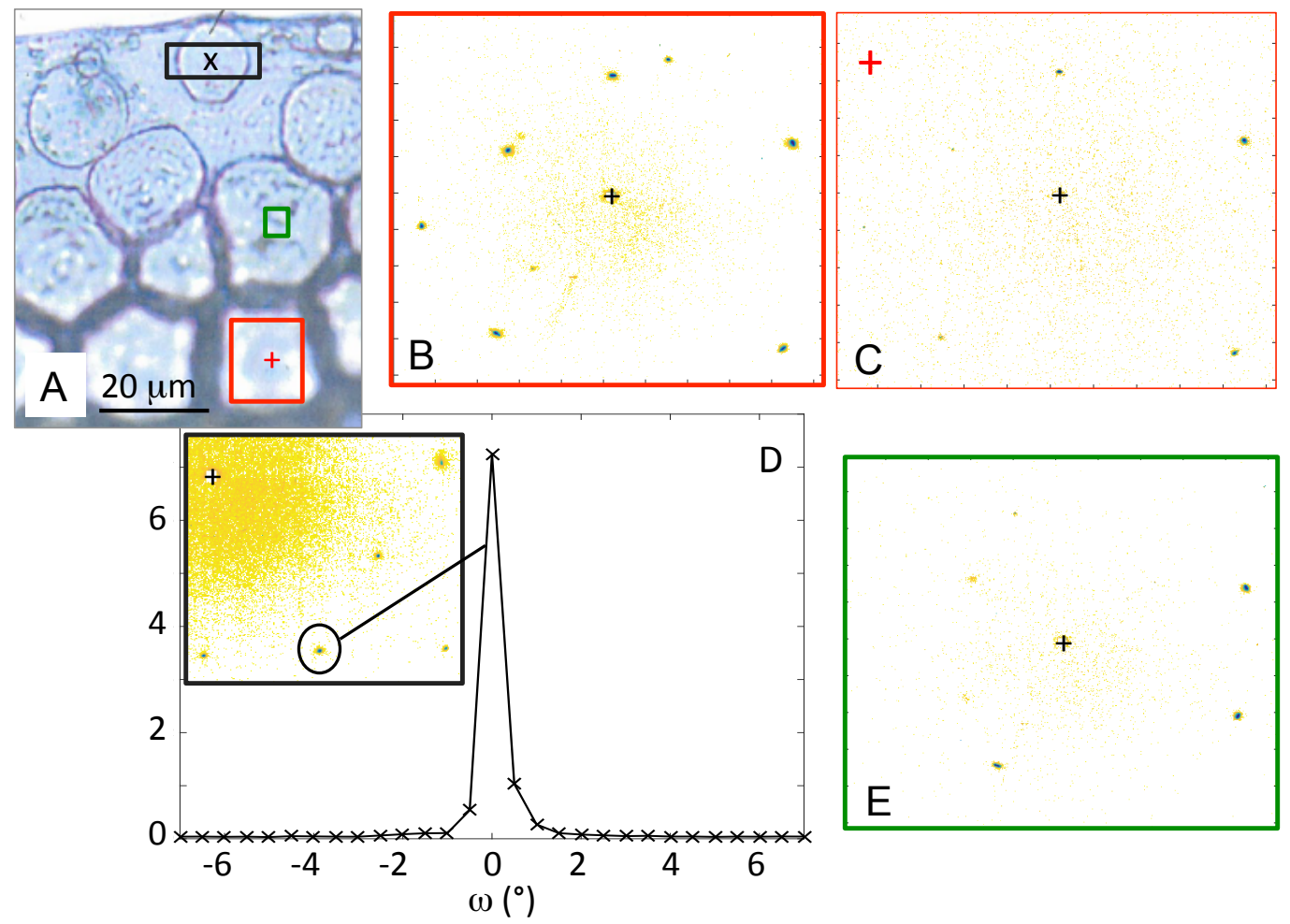

Fig. 1 (Suppl.): Single crystalline behaviour of Pinctada margaritifera prisms evidenced by X-ray Bragg micro-diffraction measurements. (A) Optical microscopy view of the shell border, showing the presence of prismatic and early prismatic (disk-like) units. The whole 
area has been characterized by $\mathrm{x}$-ray Bragg micro-diffraction. The coloured rectangles correspond to the results shown in (B)-(E). (B) 2D diffraction patterns, obtained by integrating the individual 2D patterns acquired on a whole prism (red area in (A)), at a fixed Bragg angle. The integration of the patterns preserves the sharpness of the Bragg peaks. (C) Individual 2D diffraction pattern taken at the centre of the same prism (red "+" in (A)). The strong similarity between (B) and (C) shows the homogeneous behaviour of the crystal at this scale. (D) Rocking curve performed on an early prism unit (black " $x$ " in (A)), corresponding to the Bragg peak shown in the inset (black area in (A)). The limited broadening of the peak (of about $1^{\circ}$ ) shows the overall good quality of the single crystal. (E) Same measurement as in (B) for another prism (green area in (A)). The 2D diffraction pattern shows characteristics similar to those in (B), exhibiting however a different crystalline orientation. In (B)-(E), the centre of the direct beam is indicated with the black "+".

\section{2 - Pinctada margaritifera shell edge imaged with Coherent Anti-Stokes Raman Scattering microscopy}

At the prism scale, structural investigation has been performed with 3D Coherent Anti-Stokes Raman Scattering (CARS) microscopy ${ }^{1}$ (Supplementary Figure 2).

This optical spectro-microscopy was used to image the crystalline calcite carbonate $\mathrm{C}-\mathrm{O}$ breathing mode ${ }^{2}$ at $1084 \mathrm{~cm}^{-1}$ following a scheme similar to the already described one. ${ }^{3} \mathrm{~A}$ mode-locked Nd:YVO4 MOPA laser (Plecter, Coherent, $80 \mathrm{MHz}$ repetition rate) delivers synchronous $7 \mathrm{ps}$ pulses at $1064 \mathrm{~nm}$ and $532 \mathrm{~nm}$. The beam at the doubled frequency is used to pump two OPOs (APE Levante OPO), which generate the tunable pump and Stokes pulses with a maximal tuning range of 730-960 $\mathrm{nm}$. The pump is composed of a fixed wavelength of $730 \mathrm{~nm}$ and a Stokes wavelength set at $792.8 \mathrm{~nm}$. The two beams overlap in time and space and are focused using a water immersion objective (40x, NA 1.15, CFI Apo Lambda S LWD, Nikon). The image acquisition is performed using galvanometric scanning mirrors $(6200 \mathrm{H}$, Cambridge Technology) ${ }^{4}$. Typically, the pixel dwell time is $40 \mu \mathrm{s}$ and the average powers at the focal spot are about $5-15 \mathrm{~mW}$. The forward emitted light is collected using a long working distance air objective (40x, NA 0.6, LUCPLFLN, Olympus) and focused on a channel photomultiplier tube (Hamamatsu, H10682). The laser pulse duration, of around $7 \mathrm{ps,} \mathrm{induces}$ a $5 \mathrm{~cm}^{-1}$ spectral resolution. The lateral and axial resolutions were measured to be $350 \mathrm{~nm}$ and $2.5 \mu \mathrm{m}$, respectively.

At the prism level, the carbonate concentration is heterogeneous along the prism surface (density fluctuations of about $10 \%$ ) while it presents a longitudinal spatial coherence over the shell thickness. 
A

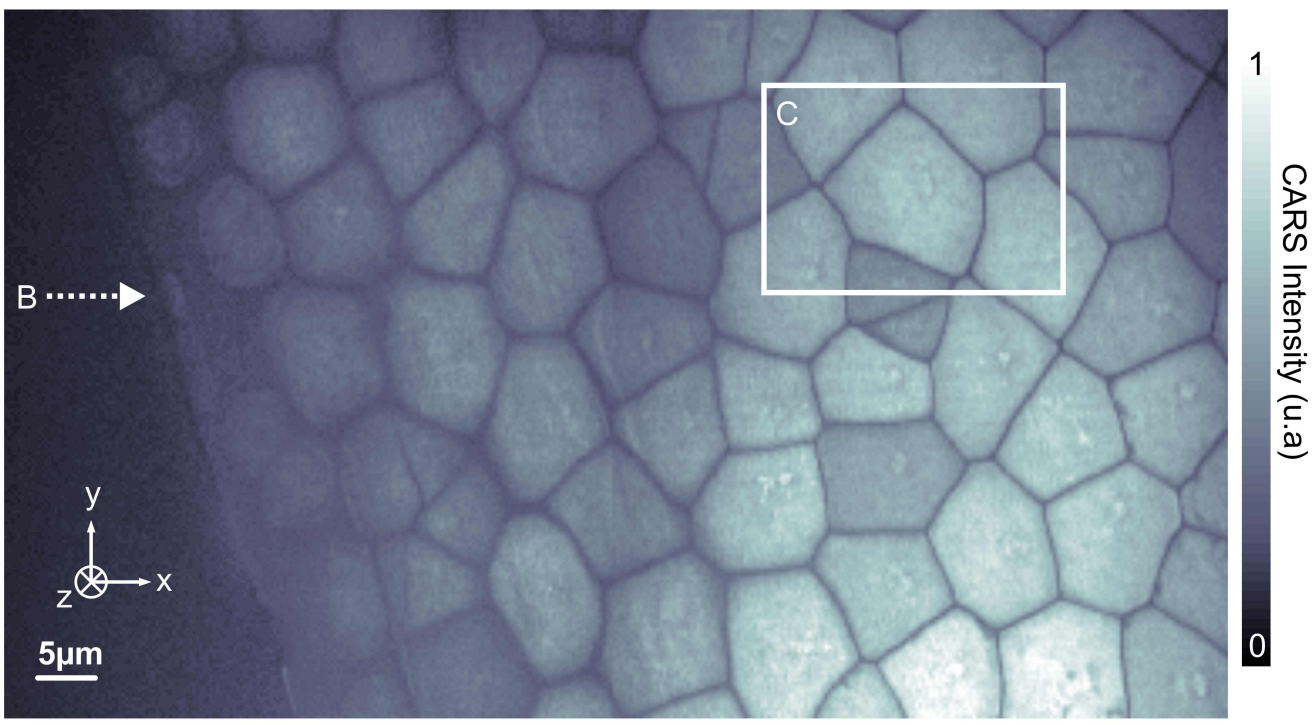

B

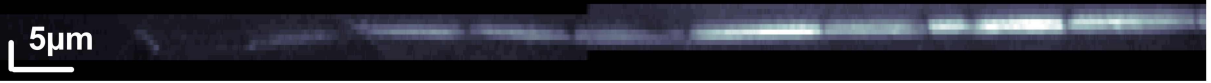

C

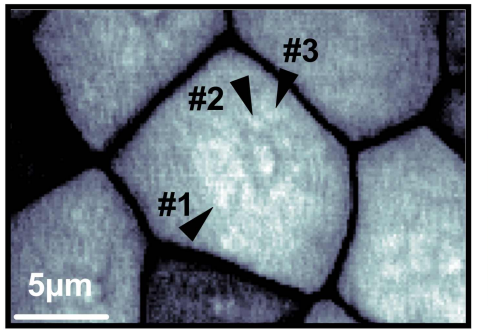

D

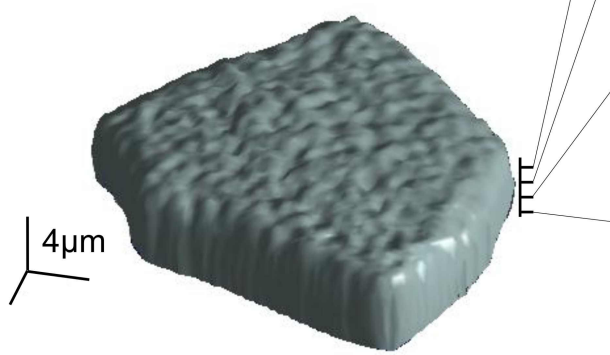

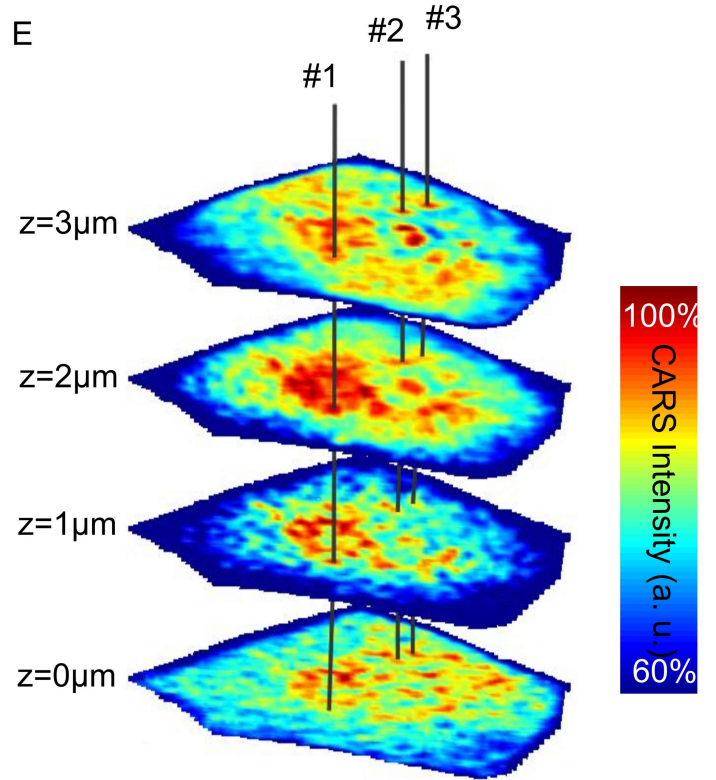

Fig. 2 (Suppl.): Pinctada margaritifera shell edge imaged with Coherent Anti-Stokes Raman Scattering (CARS) microscopy. (A) Top view resulting from the sum of the 3D CARS intensity stack along $z$. The targeted vibrational bond is the crystalline carbonate symmetric stretching mode $v_{1}$, at $1084 \mathrm{~cm}^{-1}$. (B) Longitudinal cut extracted from (A), see arrow. (C) Zoom-in of the region depicted by the rectangle on (A). The spatial distribution of CARS intensity summed over the $\mathrm{z}$ direction shows heterogeneity of the carbonate concentration at the prism level. (D) Isosurface rendering plot of the CARS intensity for one prism (threshold at $80 \%$ of the maximum intensity). (E) Different slices extracted from the internal CARS intensity. Each plane is individually normalized to its maximum signal value and plotted between $60 \%$ and $100 \%$, in order to exhibit the lateral spatial fluctuation of carbonate into the volume. A major part of the overconcentration of carbonate (as illustrated with the vertical lines \#1, \#2 and \#3) presents a longitudinal spatial coherence over the shell thickness, whose extent is longer than the axial resolution. The amplitude of the intensity 
fluctuations, in the range of $20 \%$ corresponds to a carbonate concentration fluctuation of about $10 \%$.

\section{3 - 3D Bragg micro-diffraction investigation of a Pinctada margaritifera prism}

The detailed investigation of the crystalline properties within a single prism has been performed using 3D Bragg diffraction, with a synchrotron beam focused down to of $0.3 \times 0.2$ $\mu^{2}$ ( $\mathrm{H} \mathrm{x} \mathrm{V}$, full width at half-maximum, see Methods and Supplementary Figure 3). The spatial dependency of the 3D intensity distribution at the 006 Bragg peak was recorded, in a transmission mode, by scanning the prism across the beam with translation steps of 5 and 1 $\mu \mathrm{m}$ along $\mathbf{x}$ and $\mathbf{y}$, respectively, while the $3 \mathrm{D}$ patterns were collected with an angular step of $0.1^{\circ}$ (for a total angular range of $4^{\circ}$ ). At this scale, the 3D Bragg peaks exhibit strong spatial fluctuations evidencing a non-homogeneous behavior of the crystalline properties inside the prism. Moreover, the Bragg peaks systematically show a shape elongated along a single axis contained into the $\left(\mathbf{q}_{2}, \mathbf{q}_{3}\right)$ plane. These correspond to local rotations of the crystal around an axis perpendicular to both the 006 Bragg peak and the axis describing the intensity elongation. 

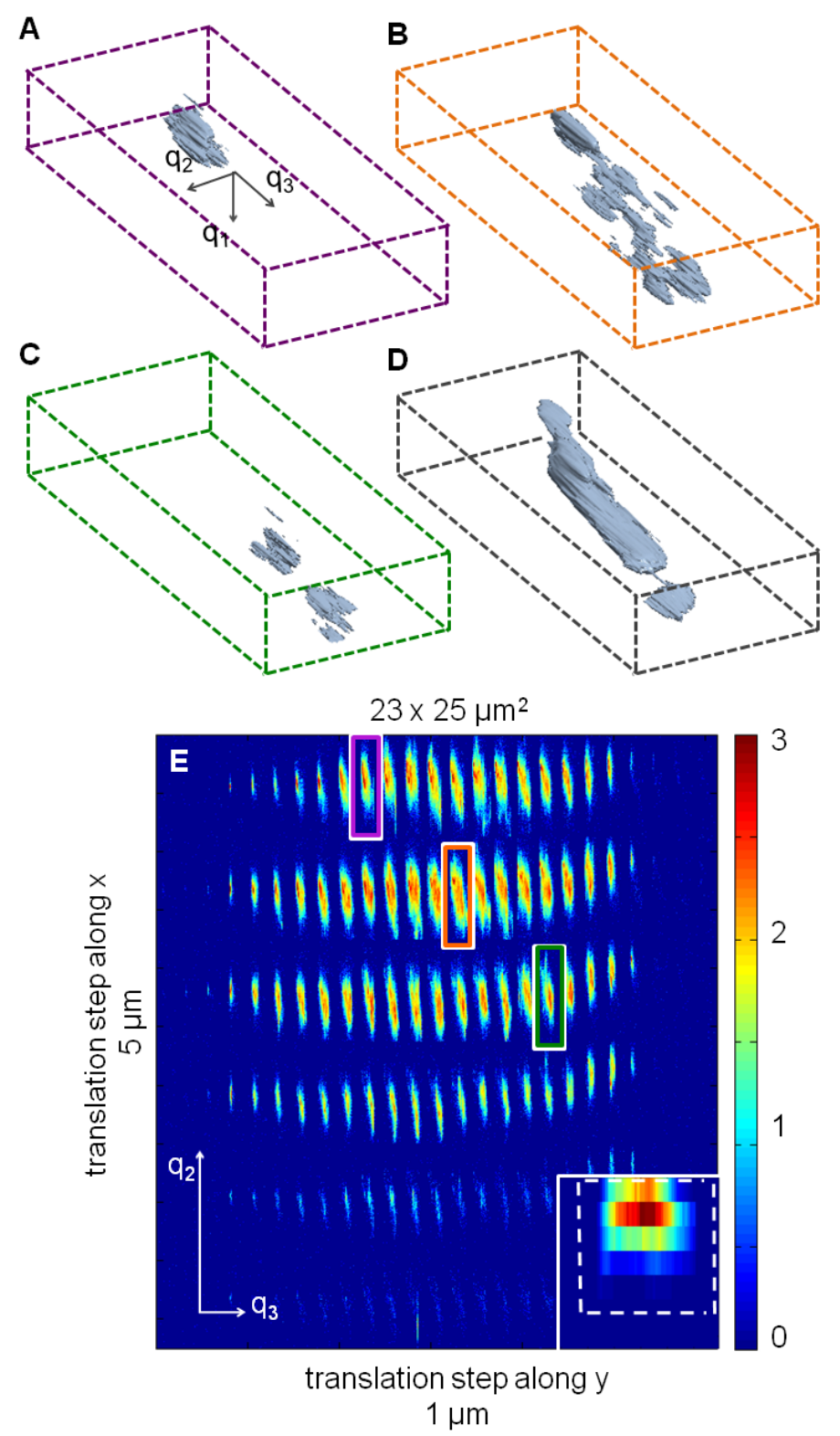

Fig. 3 (Suppl.): 3D Bragg diffraction intensity - microdiffraction experiment in Bragg geometry. (A-C) Iso-surface rendering of the 006 3D Bragg diffraction intensity pattern measured at three different illumination positions on the prism. The intensity threshold is set to 5 photons. (D) Same plot resulting from the spatial integration of the 3D intensity patterns measured over an entire prism (threshold set to 200 photons). Note the elongated shape of the diffraction pattern in the $\left(\mathbf{q}_{2}, \mathbf{q}_{3}\right)$ plane. (E) 2D intensity patterns resulting from the integration of the $3 \mathrm{D}$ Bragg peak along the $\mathbf{q}_{1}$ direction and plotted as a function of their respective illumination positions along the prism surface, i. e., $\mathrm{y}$ and $\mathrm{x}$. Positions corresponding to Figs. A-C are indicated by coloured rectangles. The length of the reciprocal space axis $\mathrm{q}_{2}$ and $\mathrm{q}_{3}$ corresponds to $1 \mathrm{~nm}^{-1}$. Inset: Totally integrated intensity plotted in the $(\mathrm{y}, \mathrm{x})$ plane. 


\section{4 - 3D Bragg ptychography: from data acquisition to image analysis}

This last section brings additional details on the 3D Bragg ptychography procedure, a newly developed microscopy approach. It is used herein to provide the detailed 3D description of the biomineral crystal. Previous works have shown that spatial resolution of a few tens of nanometres, strain sensitivity of a few $10^{-4}$ and lattice rotation sensitivity of $0.005^{\circ}$ are reachable ${ }^{5}$. This section covers many aspects of the Bragg ptychography procedure: experiments, preliminary data analysis, inversion procedure, and spatial resolution.

Illumination function: Supplementary Figure 4 presents the reconstruction of the illumination complex-valued function using the method described in a previous article. ${ }^{6}$ This information, collected before the Bragg ptychography experiment itself, is mandatory in order to optimize the scanning step sizes. In addition, it is further used during the ptychography inversion procedure, in order to calculate the $3 \mathrm{D}$ probe illuminating the sample, which is kept fixed during the image reconstruction. Note that the transverse beam size at the sample position is hereby estimated to $0.08 \times 0.1 \mu \mathrm{m}^{2}$ ( $\mathrm{H} \mathrm{x} \mathrm{V}$, full width at half-maximum).
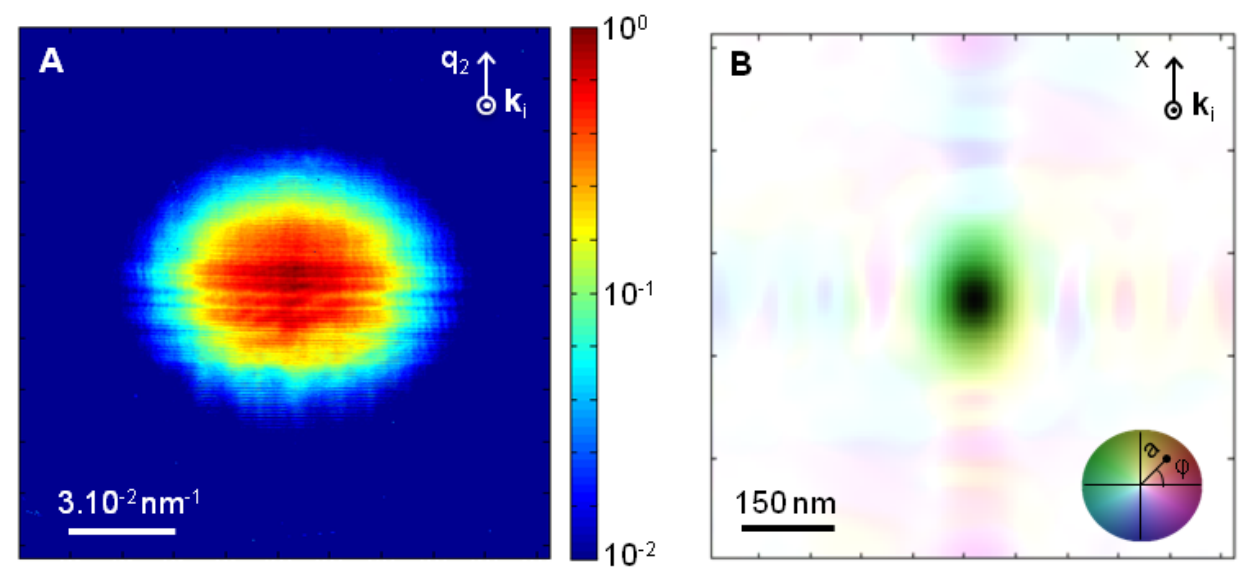

Fig. 4 (Suppl.): Imaging the illumination complex-valued function. (A) Far-field diffraction pattern of the freely propagating focused direct beam. (B) Cross section of the complex-valued wave field at the focus, retrieved using the method described in a previous article. $^{6}$

Bragg ptychography raw data: Supplementary Figure 5 presents some raw data extracted from the Bragg ptychography data set used to produce Figs. 3 and 4 (see main text). Due to the limited amount of scattered photons (the maximum intensity level is a few tens of photons/pixel), only a limited amount of speckles is visible, each of them extending over 1020 pixels (i. e., 1.6-3.2 $10^{-2} \mathrm{~nm}^{-1}$ ), in agreement with the size of the far-field direct beam (Supplementary Figure 4). The visibility is rather difficult to estimate from the data set, as a consequence of the presence of the crystalline displacement field and of the photon shot noise. However, in the inset of Supplementary Figure 5B, we produce an integrated intensity cross-section, exhibiting a visibility of about $80 \%$. 

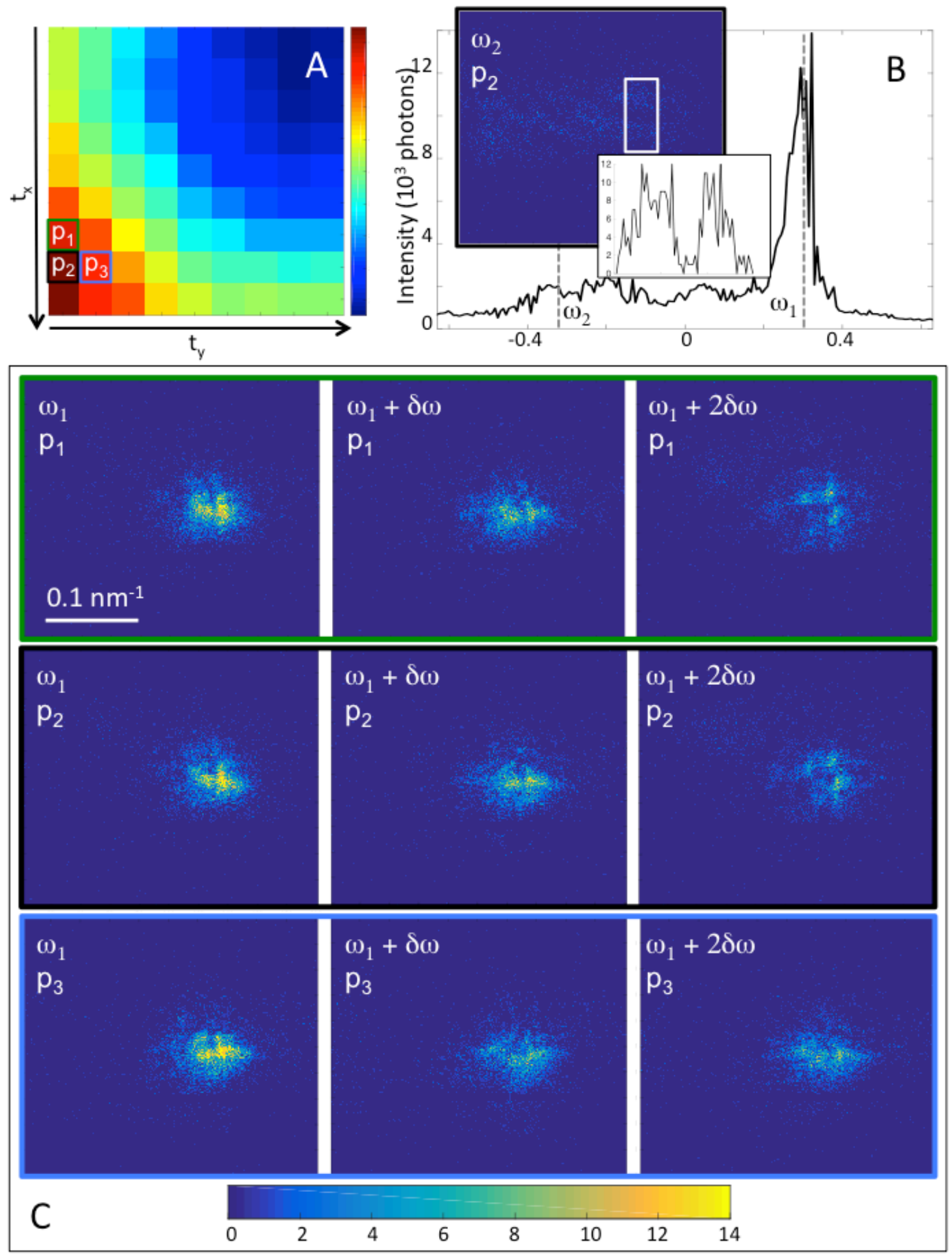

Fig. 5 (Suppl.): Bragg ptychography coherent diffraction patterns. (A) 2D plot of the Bragg diffraction intensity (110 reflection), integrated along the rocking curve, at each position of the $t_{x}$ and $t_{y}$ Bragg ptychography scans (see Methods). Color scale is linear. Three selected positions $\left(\mathrm{p}_{1}, \mathrm{p}_{2}\right.$ and $\left.\mathrm{p}_{3}\right)$ are indicated. (B) Extracted rocking curve ( $\omega$ scan in degree) at $\mathrm{p}_{2}$. Each point corresponds to the integration of one detector frame. Two selected rocking curve angles $\left(\omega_{1}\right.$ and $\left.\omega_{2}\right)$ are indicated. The $2 \mathrm{D}$ pattern obtained at $\mathrm{p}_{2}$ and $\omega_{2}$ is shown in the inset, together with an integrated intensity cross section extracted from the white rectangle 
area. (C) Series of 2D coherent diffraction patterns obtained at the $\mathrm{p}_{1}, \mathrm{p}_{2}$ and $\mathrm{p}_{3}$ positions, for successive steps ( $\delta \omega$ and $2 \delta \omega$ from $\omega_{1}$ ) along the rocking curve. Note the continuity of the signal along both sample-scanning and rocking-curve directions. The colour intensity scale is in photons. Reciprocal space scale is given in the top left pattern of (C).

Optimization of the inversion procedure: The whole inversion procedure was first optimized following a strategy already exploited in a previous article. ${ }^{7}$ To this aim, a numerical sample was created and used to generate a complete set of Bragg ptychography data using numerical parameters equal to the experimental ones (i. e., reciprocal space pixel sizes, Bragg angles, ptychography scanning directions and step sizes, photon shot noise level). However, in order to accelerate the calculation, the pixel numbers were reduced along the detector plane, so that each numerical diffraction pattern window corresponds to $94 \times 286 \times 286$ pixels. This results in a direct space pixel size of $45 \times 15 \times 7.2 \mathrm{~nm}^{3}$ along respectively, directions 1,2 , and 3 .

The 3D sample scattering factor was designed, guided by the available structural information. The planar morphology of the prisms, as the one observed in the vicinity of the shell border, was accounted for by designing a thin layer with a thickness of $1.35 \mu \mathrm{m}$. While we could not obtain a precise initial estimate of the investigated prism thickness, preliminary scanning electron microscopy investigations have shown that typical thicknesses of near shell border prisms usually range between 1.2 and $1.9 \mu \mathrm{m}$. The sample area, covering about $4 \times 4 \mu \mathrm{m}^{2}$, was defined according to the scanned area (including the probe tails). To test the density fidelity reconstruction, an empty density part was introduced all along the sample thickness, in one of the corners of the surface plane window (Supplementary Figure 6A, B). Furthermore, as the preliminary analysis of the Bragg diffraction data set evidenced the presence of mis-oriented crystalline domains (presence of several Bragg maxima along the rocking curve, Supplementary Figure 5B), a crystalline displacement field was introduced, mimicking local strain and/or orientations ${ }^{5}$. To this aim, a complex-valued function was introduced to describe the sample, with amplitude and phase related respectively to the sample density and projection of the crystalline displacement ${ }^{5}$. The sample was divided into several domains, with lateral dimensions of $220 \times 290 \mathrm{~nm}^{2}$ and elongated along the thickness direction (according to CARS results and preliminary test inversions). For each domain, a phase ramp was assigned, expressed as a linear function of one or several spatial coordinates (Supplementary Figure 6C, D). Indeed, a finite crystalline strain can be written as a linear phase ramp along the spatial direction parallel to the Bragg vector (resulting in the motion of the Bragg peak along the reference Bragg vector) while a small crystalline rotation can be approximated by a linear phase ramp along the direction perpendicular to the Bragg vector and the rotation axis (and resulting thereby in the motion of the Bragg peak along the plane perpendicular to the Bragg vector). ${ }^{5,8}$ Each domain was assigned a constant strain and/or rotations (with randomly chosen values), which could be recovered from the spatial derivatives of the phase map (Supplementary Figure 6E, F). Finally, the full complex-valued object was transformed from the laboratory frame to a non-orthogonal frame, derived from Fourier conjugation relations applied to the detection frame ${ }^{6}$. The $9 \times 93 \mathrm{D}$ diffraction patterns were calculated and further corrupted by photon shot noise (Supplementary Figure $6 \mathrm{M})$. The whole data set was used to test and optimize the inversion procedure.

As shown in a previous article ${ }^{5}$, the Bragg ptychography inversion procedure requires the introduction of a penalization term, aiming at softly constraining the reconstruction within a reasonable volume, delimited herein by the film thickness. In order to adjust the regularization parameter $\mu$, a series of inversion tests were performed using the ordered subset inversion strategy together with the Gaussian shot noise model. ${ }^{9}$ Different support thickness 
were introduced as a regularization function. The resulting error metrics were analysed (Supplementary Figure $6 \mathrm{~N}$ ) in order to optimize $\mu$, finally fixed to $10^{2}$. From this, a second series of inversion were performed in order to confirm the possibility to retrieve the sample thickness from the behaviour of the error metric. As shown in Supplementary Figure 6O, an optimum value of $1.4 \mu \mathrm{m}$ could be retrieved, for a nominal value of $1.35 \mu \mathrm{m}$, without additional structural information. The corresponding retrieved image is shown in Supplementary Figure 6(G-L). This approach was applied to the experimental data set to guess the prism thickness (Supplementary Figure 6P), estimated finally to about $1.75 \mu \mathrm{m}$.
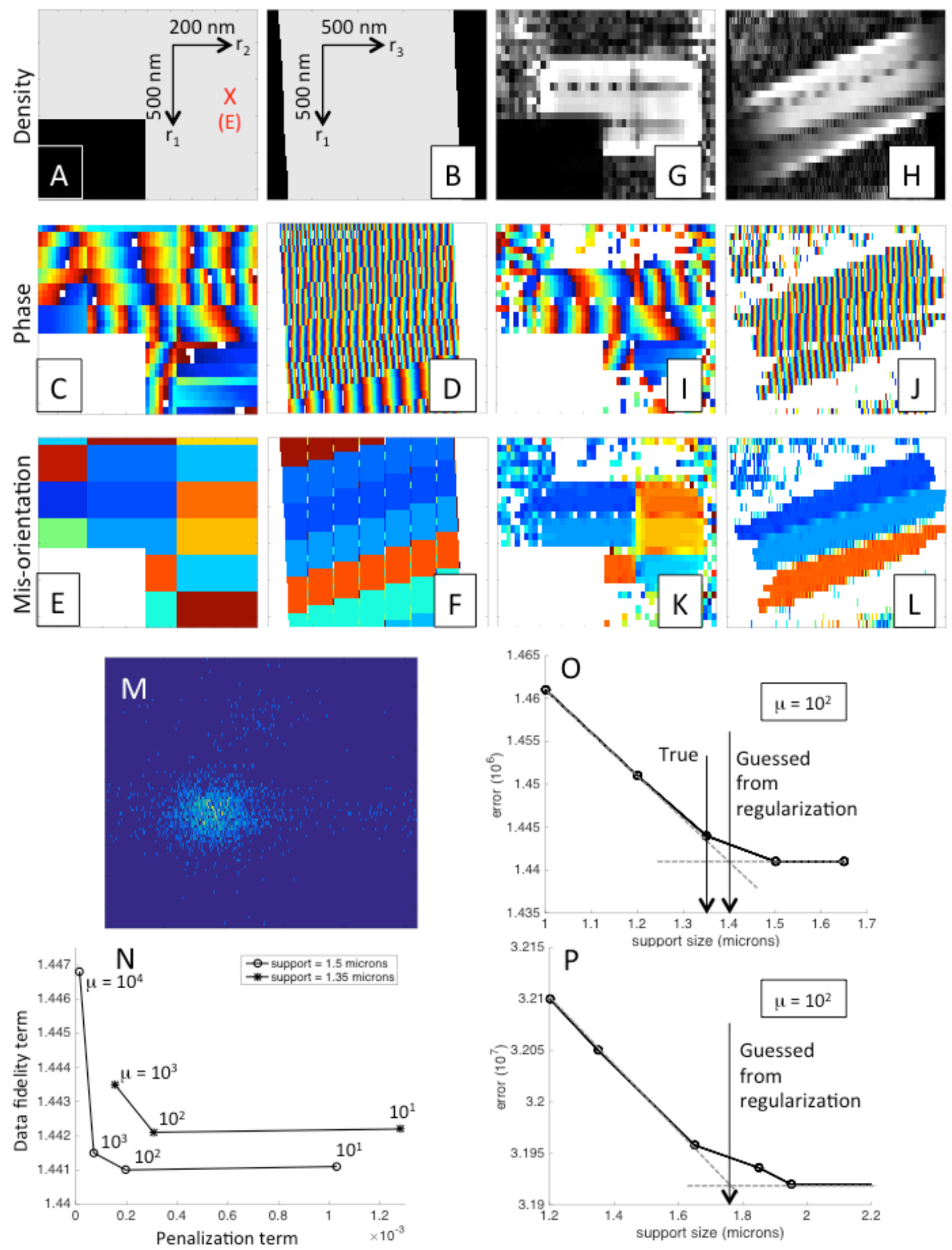

Fig. 6 (Suppl.): Numerical tests performed to optimize the inversion strategy. (A, B) Amplitude of the 3D numerical object, plotted along two different planes. (C, D) Similar plots 
of the numerical object phase. The domains of linear phase ramps correspond to domains of constant strain and mis-orientation. (E, F) Mis-orientation maps (arbitrary units), extracted from the derivative of the $3 \mathrm{D}$ phase map. $(\mathrm{G}, \mathrm{H})$ Retrieved density, obtained with the regularization parameter $\mu$ set at $10^{2}$ and a thickness support of $1.4 \mu \mathrm{m}$. Note that the density, only retrieved in a small volume corresponding to the probed region, presents a structured shape. (I, J) Retrieved phase maps. The phase ramps are well reproduced, with however arbitrary phase shifts between the domains (due to the lack of intensity between the corresponding noise corrupted Bragg peaks). (K, L) Retrieved mis-orientation maps, extracted from the derivative of the 3D phase map. Their comparisons with (E, F) show satisfying agreement and validate the inversion procedure. (M) One of the diffraction patterns, corresponding to the red-cross position in (A). (N) Data fidelity term as a function of the penalization term, obtained for several values of $\mu$ and support thickness. The inflection point corresponds to the selected $\mu$ value. (O) Estimation of the sample thickness from the behaviour of the error metrics as a function of the thickness support. (P) Same analysis performed on the experimental data set, demonstrating an optimum prism thickness of 1.75 $\mu \mathrm{m}$. 
Bragg ptychography reconstruction of a second volume from the same prism: The same procedure as the one used to obtain Figs. 3 and 4 (main text) was applied to a second Bragg ptychography data set. The result of the reconstruction is shown in Supplementary Figure 7.

A $2^{\text {nd }}$ investigated volume

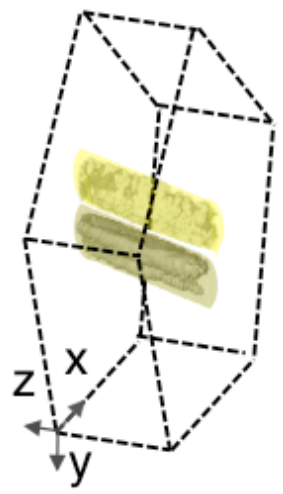

B Crystalline rotation

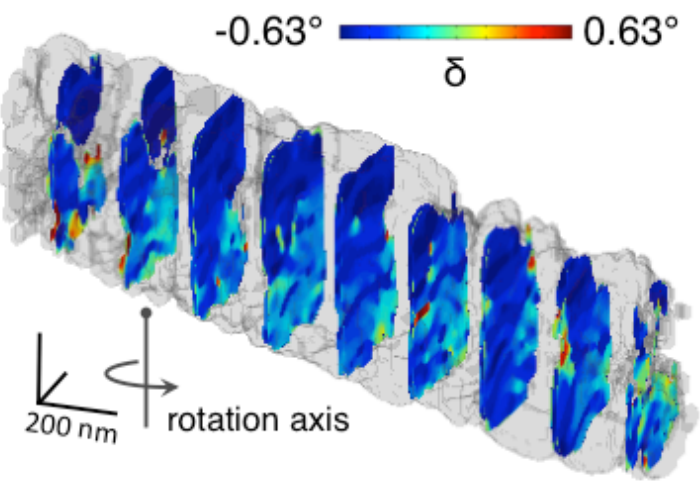

C Relative (110) strain

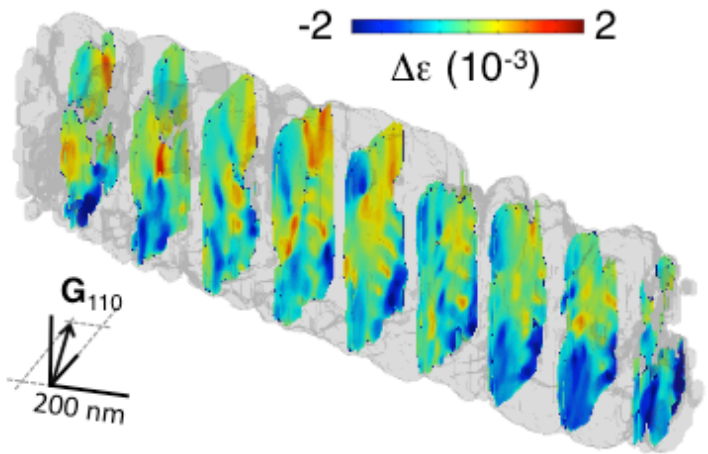

D Crystalline coherence

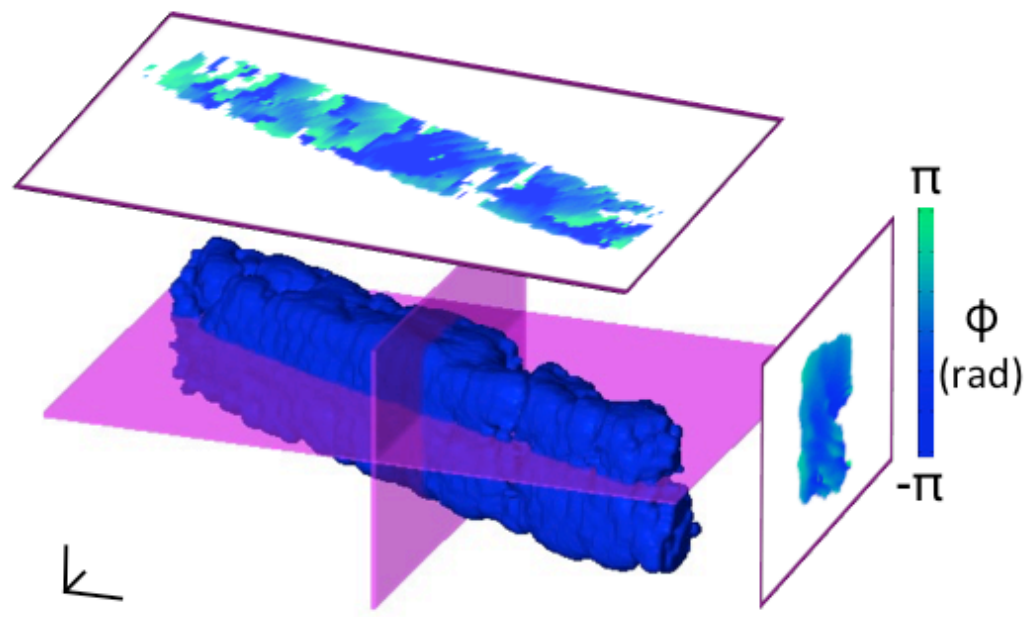

Fig. 7 (Suppl.): 3D Bragg ptychography imaging of a second region from the same Pinctada margaritifera prism. (A) Schematic view of the reconstructed prism volume together with the probed region, schematized by the yellow-gray cylinder. This region is located $2 \mu \mathrm{m}$ away from the volume presented in the main text, schematized here by the dark gray-yellow cylinder underneath the yellow cylinder. (B) and (C) Crystalline rotation $(\delta)$ and relative strain $(\varepsilon)$ variation maps shown on several planes along the reconstructed volume, corresponding respectively to the rotation of the (110) crystalline planes around the rotation axis shown in (B) and to the relative crystalline displacement along $\mathbf{G}_{110}$. (D) $2 \mathrm{D}$ cuts of the 
retrieved phase map extracted from the iso-oriented domain shown in (B). The abrupt changes visible in the phase maps correspond to the edges of the coherent crystalline domains. In average, the phase shift is about 4.4 radians, corresponding to a crystalline displacement of about $0.17 \mathrm{~nm}, i . e . \approx 2 / 3$ of the (110) lattice spacing. In (B), the 3D length scale is indicated and represents $200 \mathrm{~nm}$.

Spatial resolution estimation: The extent of the reciprocal space data set is given by $n_{j} \delta q_{j}$, with $n_{j}$ the number of pixels of the data set window, $\delta q_{j}$ the reciprocal space pixel size and $j=$ $\{1,2,3\}$. For Figs. 3 and 4 , it corresponds to $n_{1}=486, n_{2}=516, n_{3}=286$ and $\delta q_{1}=1.617 \mu m^{-}$ ${ }^{1}, \delta \mathrm{q}_{2}=1.617 \mu \mathrm{m}^{-1}, \delta \mathrm{q}_{3}=3.361 \mu \mathrm{m}^{-1}$, covering maximum wave-vector transfers of $786 \mu \mathrm{m}^{-1}$, $834 \mu^{-1}$ and $961 \mu \mathrm{m}^{-1}$, respectively along the three detection directions. According to the Fourier conjugation relations, it leads to a direct space voxel size $\delta r_{j}$ given by $2 \pi / n_{j} \delta q_{j}$, corresponding here to $\delta r_{1}=8.0 \mathrm{~nm}, \delta r_{2}=7.5 \mathrm{~nm}$ and $\delta r_{3}=6.6 \mathrm{~nm}$.

However, the voxel size of the retrieved image does not consist in an estimation of the spatial resolution, as it does not take into account $(i)$ the limited extent of the intensity signal into the data set window and ( $i i)$ the performances of the inversion process. Additionally, the Bragg diffraction modality imposes to further consider the sparsity of the data set, which results from the existence of several Bragg peaks (one for each iso-oriented domain), each of them showing a finite extent. Finally, the measurement procedure and the 3D intensity distribution being potentially anisotropic, a 3D spatial resolution has to be estimated. Point $(i)$ is illustrated in Supplementary Figure 8 (A and B), where the intensity distribution, integrated along all ptychography positions, is plotted in the vicinity of the Bragg peak corresponding to the orange mis-oriented domain (shown in Figs. 3 and 4, main text). A spatial resolution, defined by a 2-photon threshold, is found at about $35 \mathrm{~nm}$. Accounting for (ii) is more problematic: indeed, the often used Fourier shell correlation approach can not be applied here, as the narrowness of our data set does not allow to extract two still-converging sub-data sets. In Supplementary Figure 8(B and C), we present an alternative approach based on the ratios between the square of the reconstructed amplitude and the experimental intensity, integrated along all ptychography positions. A threshold of $10 \%$ leads to a 3D spatial resolution of about $48 \times 62 \times 54 \mathrm{~nm}^{3}$, along the three directions. However, this approach minimizes the agreement between two successive positions. A third estimation of the $3 \mathrm{D}$ spatial resolution is obtained directly from the retrieved image, using the thickness of the boundary between two adjacent mis-oriented domains (Supplementary Figure 8(E-H)). As a conclusion, we estimate that a value of about $40 \pm 10 \mathrm{~nm}$ along the three directions represents a fair estimation of the spatial resolution.

Finally, Supplementary Figure $8(\mathrm{E}-\mathrm{H})$ indicates the presence of fluctuations of the misorientation angle value, whose amplitudes are smaller than the mis-orientation mismatch between two domains. They result in the corrugated nature of the domain surface observed in Figs. 3 and 4 (main text). While this is clear that those fluctuations arise from the limited signal-to-noise ratio of the data, we think that they may originate from a physical structure within the sample, such as the granular arrangement. A full understanding of this effect would require additional data set with higher signal-to-noise ratio. 

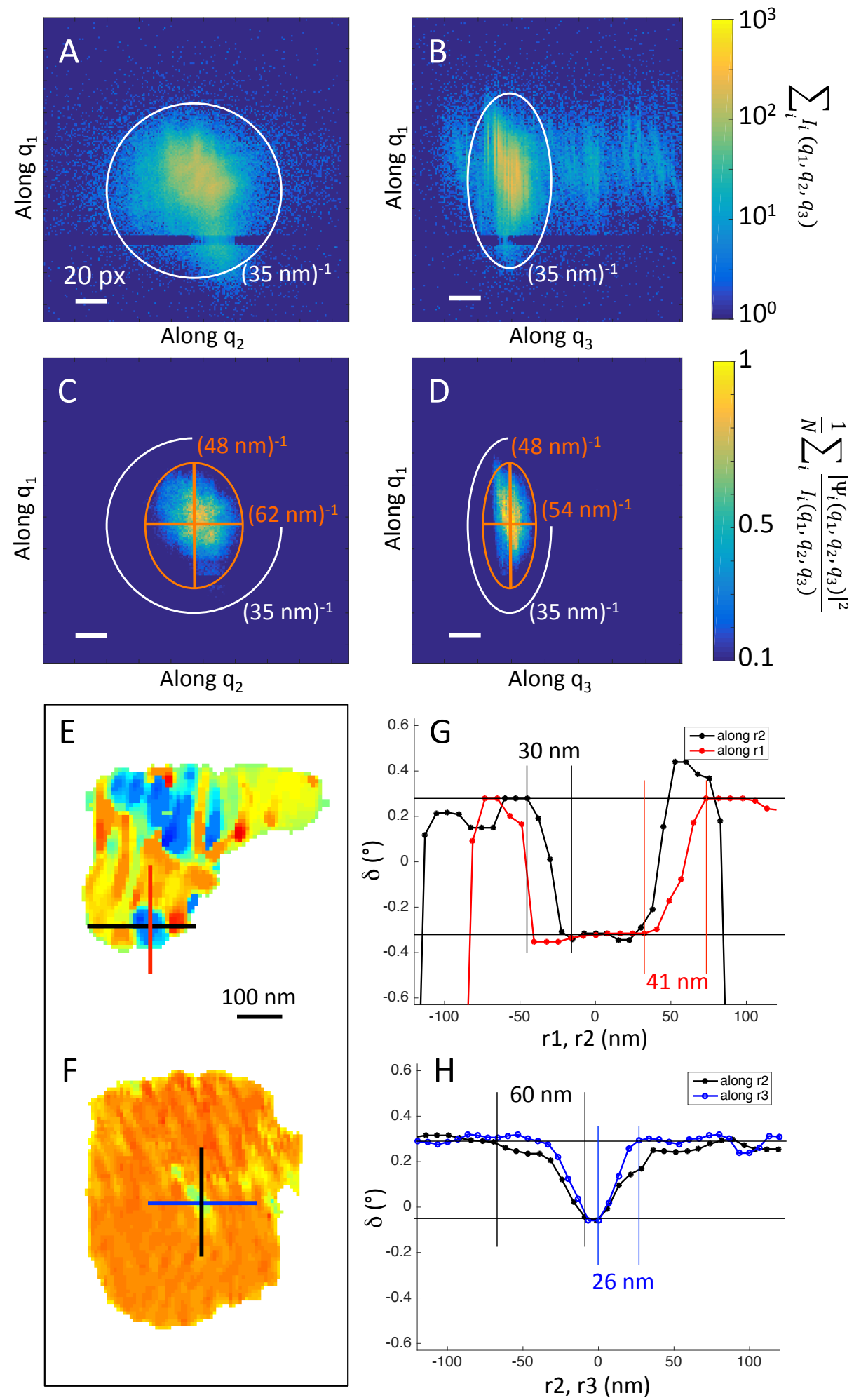

Fig. 8 (Suppl.): Estimations of the 3D spatial resolution. (A, B) 2D cross sections of the intensity distribution, integrated along the $\mathrm{N}=81$ ptychography positions $i$ and plotted in the vicinity of the Bragg peak of the $\delta=0.29^{\circ}$ mis-oriented domain (domain plotted in orange in Figs. 3 and 4, main text). The white circle, corresponding to a threshold of about 2 photons, has a diameter of $2 \pi /(35 \mathrm{~nm})$. Colour scale is in photons. (C, D) $3 \mathrm{D}$ distribution of the ratio between the square of the retrieved amplitude $\psi_{i}$ and the experimental intensity $\mathrm{I}_{\mathrm{i}}$, integrated 
along the ptychography positions $\mathrm{i}$ and normalized. The orange ellipses, corresponding to a classical threshold of $10 \%$, present long and short diameters of $2 \pi /(\mathrm{R})$, with $\mathrm{R}$ values indicated on the figures. Colour scale is in arbitrary unit. For (A-D), the reciprocal space scale is in pixels. (E, F) 2D cross sections of the retrieved rotation image, taken along 2 different planes (in the detection conjugated space). The presence of a well-defined domain is used to extract the domain boundary thickness, further plotted in $(\mathrm{G})$ and $(\mathrm{H})$. Colour scale is the same as in Supplementary Figure 3B.

\section{Bibliography}

1. Cheng, J.-X. \& Xie, X. S. Coherent Anti-Stokes Raman Scattering Microscopy: Instrumentation, Theory, and Applications. J. Phys. Chem. B 108, 827-840 (2004).

2. Tlili, M. M. et al. Characterization of $\mathrm{CaCO} 3$ hydrates by micro-Raman spectroscopy. J. Raman Spectrosc. 33, 10-16 (2002).

3. Brustlein, S. et al. Optical parametric oscillator-based light source for coherent Raman scattering microscopy: practical overview. J. Biomed. Opt. 16, 21106-21106-10 (2011).

4. Ferrand, P. GPScan.VI: A general-purpose LabVIEW program for scanning imaging or any application requiring synchronous analog voltage generation and data acquisition. Comput. Phys. Commun. 192, 342-347 (2015).

5. Pateras, A. I. et al. Nondestructive three-dimensional imaging of crystal strain and rotations in an extended bonded semiconductor heterostructure. Phys. Rev. B 92, 205305 (2015).

6. Berenguer, F. et al. X-ray lensless microscopy from undersampled diffraction intensities. Phys. Rev. B 88, 144101 (2013).

7. Chamard, V. et al. Strain in a silicon-on-insulator nanostructure revealed by 3D x-ray Bragg ptychography. Sci. Rep. 5, 9827 (2015).

8. Holt, M. V. et al. Strain Imaging of Nanoscale Semiconductor Heterostructures with X-Ray Bragg Projection Ptychography. Phys. Rev. Lett. 112, 165502 (2014).

9. Godard, P., Allain, M., Chamard, V. \& Rodenburg, J. Noise models for low counting rate coherent diffraction imaging. Opt. Express 20, 25914 (2012). 